\title{
Nuclear Dicer makes the cut
}

Dicer
processes
dsRNAs ...
to promote
the formation
of hetero-
chromatin

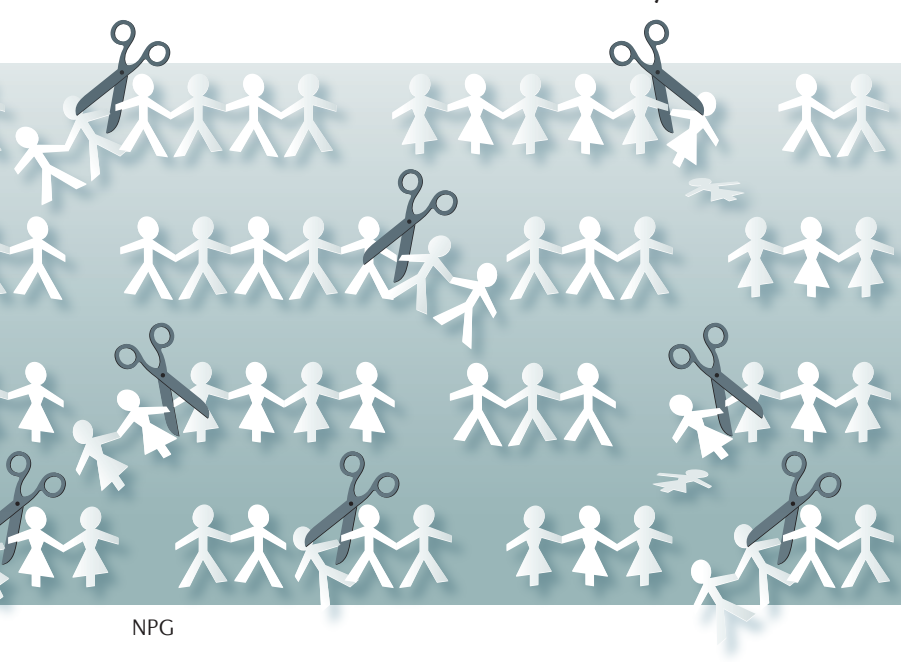

the endogenous protein localized to both the cytoplasm and the nucleus. By contrast, overexpressed GFPtagged Dicer was only detected in the nucleus of human DICER1-knockout cells, which suggests that the nuclear levels of this enzyme are tightly controlled. Dicer co-immunoprecipitated with RNA polymerase II ( $\mathrm{Pol} \mathrm{II}$ ), but this interaction was decreased in nuclear extracts that had been treated with the dsRNA-specific RNase V1; this suggests that the Dicer-Pol II association is dependent on the presence of dsRNA. Furthermore, using chromatin immunoprecipitation followed by sequencing the authors identified multiple genomic loci that were enriched in Dicer, and the addition of the transcription inhibitor $\alpha$-amanitin (which is an amatoxin) reduced Pol II and Dicer levels at four selected genes. These findings suggest that Dicer and Pol II co-associate at transcriptionally active genes.

The levels of sense and antisense transcripts of these four genes were augmented in DICER1-knockdown cells, but this increase was reduced in the presence of the RNase V1, which suggests that Dicer decreases the formation of dsRNA. The authors investigated the possibility that nuclear Dicer induces transcriptional gene silencing in mammalian cells, a process that has been described in other eukaryotic cells and involves the siRNA-AGO-mediated recruitment of methyltransferases to target chromatin to promote the generation of repressive chromatin structures. They found that Dicer-positive loci had increased levels of AGO1 and the repressive histone mark dimethylated Lys9 of histone H3 (H3K9me2), which suggests that Dicer induces the formation of $\mathrm{H} 3 \mathrm{~K} 9 \mathrm{me} 2$ through the processing of dsRNAs into siRNAs and loading of AGO1. In agreement with this, the authors found that the levels of dsRNAs and small RNAs were increased in DICER1-knockout cells and, moreover, that dsRNAs occurred at chromatin-binding sites of Dicer.

Invading viral dsRNA is eliminated through the host interferon-response pathway, which ultimately kills the infected cell; so, the authors examined whether the accumulated dsRNA in Dicerdepleted cells induces this defence mechanism. Indeed, genes encoding key proteins of the interferonresponse pathway were upregulated in DICER1-knockdown cells, which displayed increased levels of apoptosis, as evidenced by morphological abnormalities and increased annexin V labelling.

Together, the results of this study reveal a biological role for nuclear Dicer in mammalian cells. Dicer processes dsRNAs that arise during convergent transcription to promote the formation of heterochromatin possibly through AGO1-mediated recruitment of methyltransferases and the establishment of repressive histone marks.

Andrea Du Toit

ORIGINAL RESEARCH PAPER White, E. et al. Human nuclear Dicer restricts the deleterious accumulation of endogenous double-stranded RNA. Nature Struct. Mol. Biol. http://dx.doi. org/10.1038/nsmb.2827 (2014) 